\title{
Perforated gastrointestinal stromal tumor in the jejunum: A rare cause of acute abdomen
}

\author{
NAIM MEMMI ${ }^{1}$, GOKHAN CIPE$^{1}$, HUSEYIN BEKTASOGLU $^{1}$, TOYGAR TOYDEMIR $^{1}$, \\ HUSEYIN KADIOGLU ${ }^{1}$, SULEYMAN BOZKURT ${ }^{1}$, NUR BUYUKPINARBASILI ${ }^{2}$, \\ OGUZHAN KARATEPE $^{1}$ and MAHMUT MUSLUMANOGLU ${ }^{1}$
}

Departments of ${ }^{1}$ General Surgery and ${ }^{2}$ Pathology, Bezmialem Vakif University, Faculty of Medicine, Istanbul 34090, Turkey

Received May 24, 2012; Accepted August 21, 2012

DOI: $10.3892 / \mathrm{ol} .2012 .917$

\begin{abstract}
Gastrointestinal stromal tumors (GIST) are highly frequent mesenchymal tumors of the digestive tract, which mainly affect the stomach and small intestine. GISTs frequently exist with unclear symptoms. Their initial clinical presentation as acute abdomen due to their perforation is particularly rare. In the present study we report a case of a 59-year-old male presenting with acute abdomen. The final diagnosis revealed a small bowel perforation due to GIST. In this paper, we report the clinical manifestation as well as computed tomography and histopathological findings helpful for the accurate diagnosis of this rare complication of GIST. Emergency laparotomy and complete resection of tumor are essential. Following surgical resection, adjuvant tyrosine kinase inhibitor therapy should be considered for prevention of early recurrence.
\end{abstract}

\section{Introduction}

Gastrointestinal stromal tumors (GIST) are mesenchymal tumors of the digestive tract that originate from interstitial Cajal cells and account for $0.1-3 \%$ of all gastrointestinal tumors. They are usually located in the stomach and small intestine (1), but they can be located anywhere in the gastrointestinal tract, including the omentum and peritoneum. Generally, GISTs have a silent behavior and are diagnosed incidentally. Approximately $40 \%$ of GIST cases cause intestinal bleeding (2). Perforation is rarely observed in GISTs; however, we present a case of perforated GIST located in the jejunum as a rare cause of acute abdomen.

Correspondence to: Dr Gokhan Cipe, Department of General Surgery, Bezmialem Vakif University, Faculty of Medicine, Adnan Menderes Bulvar, Istanbul 34090, Turkey

E-mail: gokhancipe@hotmail.com

Key words: gastrointestinal stromal tumor (GIST), perforation, small intestine

\section{Case report}

A 59-year-old male was admitted to the emergency department of the Bezmialem Faculty of Medicine Hospital with acute abdominal pain during the previous $20 \mathrm{~h}$. The patient had no complaint of nausea or vomiting; however, the patient did have a history of diabetes mellitus type II and had undergone a coronary artery bypass surgery 6 years previously. On admission, the patient's vital signs were stable, while his physical examination revealed abdominal distention, generalized tenderness and guarding. No palpable mass was revealed on physical examination due to abdominal guarding, and bowel sounds were hypokinetic. Blood cell count was 9,800 cells $/ \mu 1$ (normal range, 4,000-11,000 cells/ $\mu \mathrm{l}$ ) and the C-reactive protein value was $6.5 \mathrm{mg} / \mathrm{dl}$, which was 13 times the upper range (normal range, $<0.5$ ).

A contrast enhanced computed tomography (CT) scan revealed a $12 \times 10 \times 9-\mathrm{cm}$ mass located at the level of the pelvic inlet on the midline posterosuperior to the urinary bladder with a solid character containing centrally necrotic areas and well-vascularized periphery (Fig. 1). Free fluid was identified around the mass, and nearby intestinal structures were inflamed. A laparotomy was conducted immediately. Subsequently a $12-\mathrm{cm}$ diameter mass was identified in the jejunum located $150 \mathrm{~cm}$ from the Treitz's ligament, and a small perforation area was observed at the medial side of the mass (Fig. 2). Generalized free fluid colored by bile was detected in the abdomen, while fibrinous inflammation and pseudomembranous colitis were identified around the mass, particularly on the intestinal structures in the pelvis. Segmentary jejunal resection, including the mass lesion, with clear macroscopical margins was conducted (Fig. 3). Handling was avoided to prevent the risk of having to conduct end to end anastomosis due to the dirty content of the abdomen and to maintain a safer approach to the patient. We anastomosed the posterior walls of each side and made a loop jejunostomy from the right lower quadrant. Histopathological investigation of the specimen revealed interlaced bundles of spindle-like tumor cells with high mitotic figures [7/50 high-power field (HPF)], and a $\mathrm{Ki}-67$ value of $8 \%$. Immunohistochemical results were C-KIT (Fig. 4A) and DOG1 positive (Fig. 4B), CD34 focally positive, S-100 positive, and smooth muscle actin (SMA) and desmin 
A

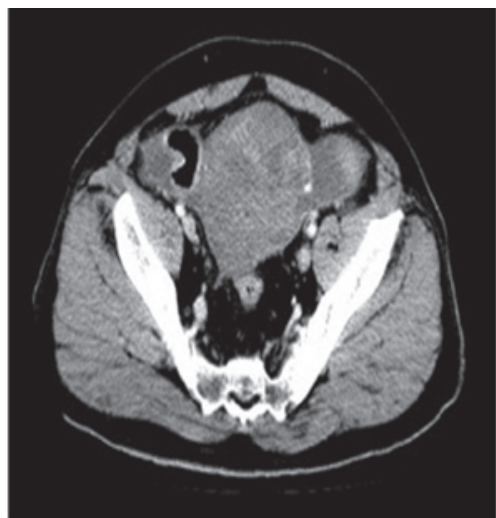

B

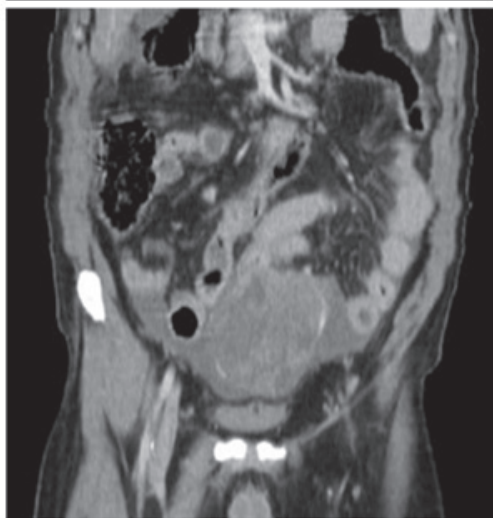

C

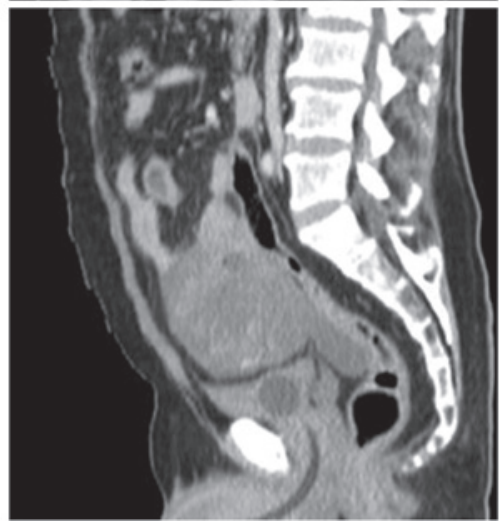

Figure 1. (A) Axial view of contrast-enhanced CT scan revealed a $12 \times 10 \times 9-\mathrm{cm}$ solid mass containing centrally necrotic areas and well-vascularized periphery. (B) Coronal and (C) sagittal views identified the mass lesion located at level of pelvic inlet on the midline posterosuperior to the urinary bladder. CT, computed tomography.

negative. As a result of these findings, the tumor was diagnosed as a GIST. A total of 6 lymph nodes were harvested, of which all were nonspecific. Surgical margins were confirmed as clear and the postoperative period was uneventful, so the patient was discharged on the 10th postoperative day.

\section{Discussion}

GIST is a visceral tumor arising from any site of the gastrointestinal tract. Approximately $60-70 \%$ of cases occur in the stomach, $25-35 \%$ in the small intestine and $10 \%$ in the jejunum, while the esophagus, colon, rectum and appendix are rarely affected (1). Approximately $10-30 \%$ of patients with GISTs may be asymptomatic. The most common symptoms associated with jejunal GISTs are vague, non-specific

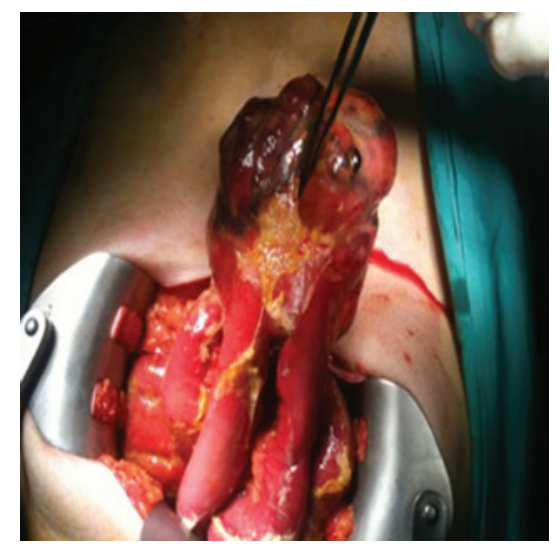

Figure 2. Approximately $12-\mathrm{cm}$ diameter mass arising from the jejunum wall with a small perforation area at the medial side.

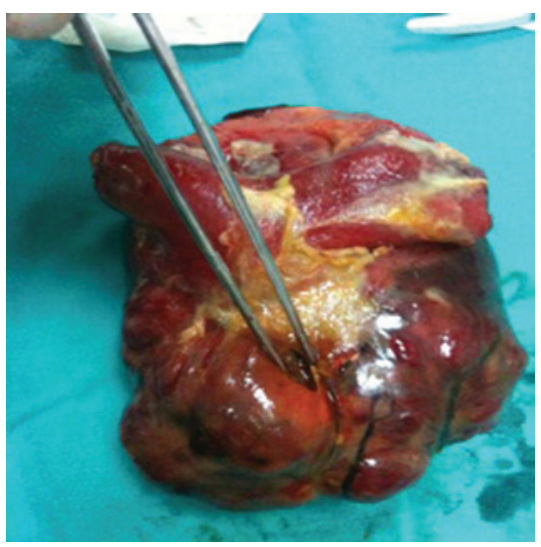

Figure 3. Perforated area following resection.

A

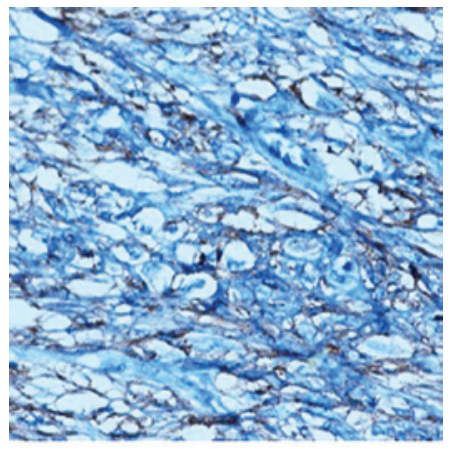

B

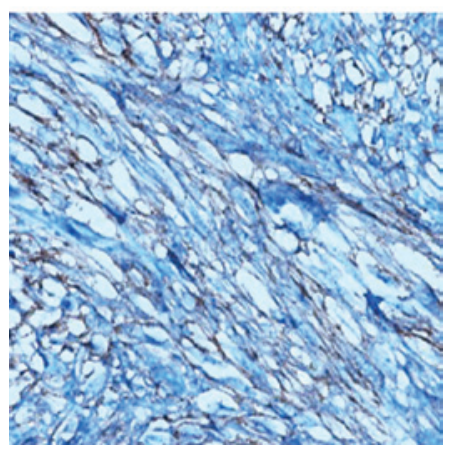

Figure 4. Immunohistochemical analysis of the tumor revealed positive expression of (A) C-KIT and (B) DOG1. 
abdominal pain or discomfort. Patients who have jejunal GIST usually suffer from abdominal pain or palpable mass, and also complain of early satiety or abdominal fullness. Jejunal GISTs may cause symptoms secondary to obstruction or hemorrhage. Pressure necrosis and ulceration of the overlying mucosa may cause gastrointestinal bleeding, and patients who experience significant blood loss may suffer from malaise and fatigue. Obstruction may result from the intraluminal growth of the tumor or luminal compression from an exophytic lesion. Fever, anorexia and weight loss are rarely observed (2) and GISTs originating from the jejunum seldom cause perforation and acute diffuse peritonitis (3-6).

It is difficult to diagnose a jejunal GIST preoperatively due to the nonspecific and variable clinical symptoms, and it is also difficult to distinguish the tumor based solely on images. Although a CT scan is a commonly offered imaging modality for patients with suspected abdominal GISTs, magnetic resonance imaging (MRI) provides better information than $\mathrm{CT}$ in the preoperative workup (7). The definitive diagnosis of the majority of jejunal GISTs is revealed by histopathological examination of the specimen. Approximately $95 \%$ of GISTs express CD117, which is part of the KIT receptor tyrosine kinase. Additionally, DOG1, a recently defined monoclonal antibody against a chloride channel protein expressed by GIST, is positively expressed in $95 \%$ of GISTs (8). DOG1 is a novel marker of GISTs as it has a higher sensitivity and specificity compared with CD34, particularly in the detection of moderate and high risk GIST. Therefore, the present case was diagnosed by immunohistochemical examination of C-KIT and DOG1 positivity.

To date, surgery is the only potentially curative therapy for patients with primary, resectable GIST. Nonmetastatic GISTs greater than $2 \mathrm{~cm}$ should be resected. A lymphadenectomy is not conducted because lymph node metastases are rare (9). Although the size of tumor in this case was large, there were no harvested positive lymph nodes in the present case. The management of GIST has undergone significant revolution over last decade. Tyrosine kinase inhibitor therapy has significantly improved overall survival in patients with advanced disease and should be continued indefinitely. Prior to the development of imatinib, recurrences were common even in patients undergoing surgery. Adjuvant imatinib for 3 years should be considered in patients undergoing resection for primary disease (10).

In conclusion, we report a case of a male with a perforated GIST in the jejunum causing acute diffuse peritonitis. The clinical outcome is worse when this tumor presents with bowel perforation and peritonitis; therefore, if an abdominal mass presents with diffuse peritonitis, the possibility of jejunal GIST perforation should be considered, even though it is extremely rare. A high degree of suspicion is necessary in view of the high morbidity rates resulting from a delayed diagnosis of the disease.

\section{References}

1. Connolly EM, Gaffney E and Reynolds JV: Gastrointestinal stromal tumors. Br J Surg 90: 1178-1186, 2003.

2. Tran T, Davila JA and El-Serag HB: The epidemiology of malignant gastrointestinal stromal tumors: analysis of 1,458 cases from 1992 to 2000. Am J Gastroenterol 100: 162-168, 2005.

3. Feng F, Chen F, Chen Y and Liu J: A rare perforated gastrointestinal stromal tumor in the jejunum: a case report. Turk J Gastroenterol 22: 208-212, 2011.

4. Karagülle E, Türk E, Yildirim E, Gõktürk HS, Kiyici H and Moray G: Multifocal intestinal stromal tumors with jejunal perforation and intra-abdominal abscess: report of a case. Turk J Gastroenterol 19: 264-267, 2008.

5. Efremidou EI, Liratzopoulos N, Papageorgiou MS and Romanidis K: Perforated GIST of the small intestine as a rare cause of acute abdomen: surgical treatment and adjuvant therapy. Case report. J Gastrointestin Liver Dis 15: 297-299, 2006

6. Özben V, Çarkman S, Atasoy D, Doğusoy G and Eyüboğlu E: A case of gastrointestinal stromal tumor presenting with small bowel perforation and internal hernia. Turk J Gastroenterol 21: 470-471, 2010

7. Amano M, Okuda T, Amano Y, Tajiti T and Kumazaki T: Magnetic resonance imaging of gastrointestinal stromal tumor in the abdomen and pelvis. Clin Imaging 30: 127-131, 2006.

8. Grover S, Ashley SW and Raut CP: Small intestine gastrointestinal stromal tumors. Curr Opin Gastroenterol 28: 113-123, 2012.

9. Fong Y, Coit DG, Woodruff JM and Brennan MF: Lymph node metastasis from soft tissue sarcoma in adults. Analysis of data from a prospective database of 1772 sarcoma patients. Ann Surg 217: 72-77, 1993.

10. Miettinen $\mathbf{M}$ and Lasota J: Gastrointestinal stromal tumors: pathology and prognosis at different sites. Semin Diagn Pathol 23: 70-83, 2006. 